\title{
Efeito da idade, do sexo, da altura e do índice de massa corporal no tempo de sucção oral de líquido
}

\author{
Effect of age, gender, height and body mass index on time of oral liquid suction
}

\section{Resumo}

Objetivo: Avaliar o efeito da idade, do sexo, da altura e do índice de massa corporal (IMC) no tempo de sucção oral de água em pessoas normais. Método: O método constou de, em duplicata, cronometrar o tempo de sucção contínua de $40 \mathrm{ml}$ de água à temperatura ambiente, utilizando um canudo com $6 \mathrm{~mm}$ de diâmetro e outro com $3 \mathrm{~mm}$ de diâmetro. Cada voluntário foi avaliado sentado, com a cabeça formando um ângulo de $90^{\circ}$ entre a mandíbula e o pescoço. Foi permitida a deglutição. Em um grupo de 15 indivíduos, foi avaliada a reprodutibilidade do teste em quatro dias diferentes. $\mathrm{O}$ trabalho incluiu 180 indivíduos, o grupo jovem (20 a 30 anos, 45 homens e 45 mulheres) e o grupo idoso (65 a 89 anos, 45 homens e 45 mulheres). Resultados: Observou-se diferença significativa no tempo de sucção entre jovens e idosos, sendo maior para os idosos do que para os jovens. Com o canudo de menor diâmetro, as mulheres idosas tiveram maior tempo de sucção do que os homens idosos. Em mulheres jovens, foi encontrada correlação positiva entre o IMC e o tempo de sucção. Não se observou influência da altura. A repetição do estudo em quatro dias mostrou o mesmo resultado. Conclusão: Concluímos que o envelhecimento compromete a sucção de líquido, e que este comprometimento é maior nas mulheres do que nos homens. Em mulheres jovens, maior IMC está associado ao maior tempo de sucção.

\section{Abstract}

Objective: To assess the effect of age, gender, height and body mass index (BMI) on oral water suction. Method: During the test, performed in duplicate, the time one person needs to continuously sip $40 \mathrm{ml}$ of water was measured at room temperature using a straw $6 \mathrm{~mm}$ in diameter and another straw $3 \mathrm{~mm}$ in diameter. The volunteers were asked
Letícia Sawamura Kubo' Weslania Viviane do Nascimento ${ }^{2}$ Roberto Oliveira Dantas ${ }^{2}$
Palavras-chave: Sucção. Distribuição por Idade e Sexo. Deglutição. Estatura. Índice de Massa Corporal.

\footnotetext{
Clínica LSK Fonoaudiologia. Ribeirão Preto, SP, Brasil.

2 Departamento de Clínica Médica. Faculdade de Medicina de Ribeirão Preto. Universidade de São Paulo. Ribeirão Preto, SP, Brasil.

Correspondência / Correspondence

Roberto Oliveira Dantas

E-mail: rodantas@fmrp.usp.br
} 
to remain seated with their head in a $90^{\circ}$ angle between jaw and neck. If necessary they could swallow. In order to verify the reproducibility of the test the same evaluation was conducted in a group of 15 individuals, four times on separate days. The investigation included 180 asymptomatic individuals, a younger group (aged 20 to 30 years, 45 men and 45 women) and an older group (aged 65 to 89 years, 45 men and 45 women). Results: Sucking time was longer for elderly than for young individuals. There was a gender difference for older subjects when the $3 \mathrm{~mm}$ straw was used, with a longer time for women than for men. Intra-subject variation was not significant. In young women BMI showed positive correlation with time of suction. Time of suction was not associated with height. Conclusion: We concluded that the ageing process causes impairment of liquid suction, and that this impairment is more intense in women than in men. In young women an increase in BMI is associated with a longer liquid suction time.
Key words: Suction.

Distribution by Age and Gender. Swallowing. Height. Body Mass Index.

\section{INTRODUÇÃO}

A sucção é um reflexo inato presente desde a vida intrauterina. É por meio dela que a criança tem os primeiros contatos com o mundo exterior, satisfazendo, além da nutrição, as necessidades afetivas. ${ }^{1}$ É controlada por um gerador de padrão central, isto é, uma rede neural localizada na formação reticular, de onde são enviados impulsos motores para a realização da função, que é efetuada com base na informação sensória para atender às demandas e necessidades funcionais. ${ }^{2}$

Fazem parte da cavidade oral os lábios, dentes, palato duro, palato mole, úvula, mandíbula, soalho da boca, língua e pilares. Para que a sucção ocorra, é necessário o bom funcionamento da musculatura labial e da musculatura oral, para evitar escape do alimento da cavidade oral e para assegurar que o material não caia nos sulcos laterais, ${ }^{3}$ e também para que seja possível criar a pressão negativa necessária para a sucção. ${ }^{4}$

Estudos revelam diferenças anatômicas e funcionais quanto ao sexo, e influência da idade na capacidade oral, na deglutição e na coordenação respiração-deglutição..$^{5-22}$ Mulheres têm trânsito orofaríngeo mais lento, ${ }^{10}$ menor fluxo orofaríngeo, ${ }^{6}$ e maior tempo de fechamento laríngeo durante a deglutição do que os homens. ${ }^{16}$ O envelhecimento causa diminuição do fluxo do material deglutido pela boca e faringe $\mathrm{e}^{7,14}$ e trânsito prolongado pela transição faringoesofágica. ${ }^{16}$ Também são observadas alterações na motilidade da transição faringoesofágica e do corpo do esôfago. ${ }^{11,12,21,22}$
Resultados prévios sugerem haver influência da idade na sucção de líquido, sendo que nos idosos a sucção forçada provoca menor pressão de sucção do que nos jovens. $^{23} \mathrm{O}$ menor volume de líquido aspirado durante a sucção foi associado ao menor tempo de sucção e menor volume intraoral..$^{23}$ Os autores utilizaram, nesta investigação, um canudo com $6 \mathrm{~mm}$ de diâmetro e concluíram que o volume do bolo obtido com a sucção está correlacionado com o diâmetro do canudo. $^{23}$ Outra publicação refere que quanto menor o diâmetro do canudo, maior a atividade muscular necessária na sucção. ${ }^{24}$ Essa atividade muscular pode perder eficiência com o processo de envelhecimento. ${ }^{25}$

Os objetivos desta investigação foram verificar: 1) se há diferença no tempo de sucção do mesmo volume de água entre jovens (20 a 30 anos) e idosos (65 a 89 anos); 2) se há diferença entre os sexos masculino e feminino; 3) a influência do diâmetro do canudo utilizado na sucção; 4) se há influência do índice de massa corporal (IMC) e da altura no tempo de sucção. Nossa hipótese é de que o envelhecimento altera a sucção de líquido e que possa haver influência do sexo, altura e IMC neste tempo.

\section{MATERIAL E MÉTODO}

A sucção foi avaliada em 180 indivíduos, de ambos os sexos, com idades variando entre 20 e 89 anos, no período de julho de 2010 a julho de 2011. Foram medidos o peso e a altura, e registrados o sexo e a idade. 
Foram excluídos da pesquisa indivíduos com obesidade grau II e III, distúrbios neurológicos, distúrbios psiquiátricos, respiradores orais crônicos, aqueles que tinham qualquer alteração anatômica ou funcional que impedisse ou alterasse o vedamento labial e a realização da sucção, pessoas com sintomas digestivos relacionados com a deglutição (disfagia, engasgo, tosse, regurgitação) e com o estômago (dor epigástrica, dispepsia, náusea, vômitos). Foram incluídos na pesquisa indivíduos com: peso normal, sobrepeso e obesidade grau I, respiração normal, doenças que não comprometiam a sucção e/ou deglutição e sem alterações nas estruturas envolvidas na sucção.

Foi considerado normal o índice de massa corporal(IMC) entre 18,5 e $24,9 \mathrm{~kg} / \mathrm{m}^{2}$; sobrepeso, IMC entre 25,0 e $29,9 \mathrm{~kg} / \mathrm{m}^{2}$; e obesidade grau I, IMC entre 30,0 e $34,9 \mathrm{~kg} / \mathrm{m}^{2}$. Obesos graus II e III foram aqueles com IMC igual ou acima de 35,0 e $40,0 \mathrm{~kg} / \mathrm{m}^{2}$, respectivamente, e não foram incluídos na investigação. O IMC foi calculado dividindo-se o peso (em quilogramas) pela altura (em metros) ao quadrado. ${ }^{26}$

Os indivíduos foram divididos em dois grupos: jovens (20 a 30 anos), 45 do sexo masculino e 45 do sexo feminino; idosos (65-89 anos), 45 do gênero masculino e 45 do gênero feminino (tabela 1).

O teste consistiu em cronometrar o tempo necessário para a sucção contínua de $40 \mathrm{ml}$ de água pura à temperatura ambiente (aproximadamente $25^{\circ} \mathrm{C}$ ), contida dentro de um tubo de ensaio transparente graduado com capacidade para $45 \mathrm{ml}$ (Siemens Medical Solutions Diagnostics, Tarrytown, NY, USA), utilizando um canudo com diâmetro de $6 \mathrm{~mm}$ e outro canudo com diâmetro de $3 \mathrm{~mm}$ (Sky, VP Indústria de Plástico Ltda, Londrina, PR). Foi solicitado ao voluntário que permanecesse sentado, com os pés apoiados no chão, com a cabeça formando um ângulo de $90^{\circ}$ entre a mandíbula e o pescoço e lábios ocluídos. Foi dito a ele: "Aspire sem interrupção toda água que está neste tubo, até nossa orientação para parar". Durante a tarefa de sucção, a pessoa poderia deglutir, mas o canudo não era retirado da boca e a tarefa continuava sem interrupção. Foi considerado início da sucção o momento em que a água começou a subir pelo canudo, e fim da sucção o momento em que terminava a água no recipiente. Foi utilizado o cronômetro Oregon Scientific modelo SL210 (Hong Kong, China). Foi medido todo o tempo de sucção, independentemente ou não da ocorrência de deglutição. Estabelecemos o volume de $40 \mathrm{ml}$ para o teste, considerando que este é um volume de líquido tolerado na cavidade oral por pessoas normais, sem a necessidade de deglutição. ${ }^{20}$

Visando a maior confiabilidade, as análises foram realizadas em duplicatas, completando, assim, um total de 160,0 $\mathrm{ml}$ de água por pessoa. A cada $40 \mathrm{ml}$ foi feito um intervalo mínimo de 60 segundos, com tempo maior se necessário. A primeira duplicata foi realizada com o canudo de $6 \mathrm{~mm}$ de diâmetro, e a segunda, com o canudo de $3 \mathrm{~mm}$ de diâmetro.

Para verificar a reprodutibilidade do teste, foram realizadas repetidas avaliações em um grupo de 15 indivíduos do grupo jovem escolhidos ao acaso, sendo que cada voluntário participou do mesmo teste quatro vezes, sendo uma semana após o primeiro teste, duas semanas após o segundo teste e quatro semanas após o terceiro teste.

A análise estatística foi feita no Centro de Métodos Quantitativos da Faculdade de Medicina de Ribeirão Preto, USP (CEMEQ). Foi utilizado o modelo de regressão linear com efeitos mistos (efeitos aleatórios e fixos). Os modelos lineares de efeitos mistos são utilizados na análise de dados em que as respostas estão agrupadas (medidas repetidas para um mesmo indivíduo) e a suposição de independência entre as observações num mesmo grupo não é adequada. ${ }^{27}$ Nesses estudos, são verificados dois tipos de variabilidade: intra e interindivíduos. A primeira é decorrente da repetição do experimento nos mesmos indivíduos, enquanto que a segunda é a variação decorrente de diferentes indivíduos, sob as mesmas condições experimentais. Ambas decorrem de variabilidade biológica e de erros de medição ou 
cálculos, porém a segunda ainda pode decorrer de diferenças entre indivíduos. $\mathrm{O}$ modelo misto utilizado para descrever o experimento estima os dois componentes de variabilidade. Tal modelo tem como pressuposto que o resíduo obtido por meio da diferença entre os valores preditos pelo modelo e os valores observados tenha distribuição normal com média 0 e variância constante. Nas situações em que tal pressuposto não foi observado, transformações na variável resposta foram utilizadas. O ajuste do modelo foi feito por meio do software SAS versão 9.0. ${ }^{28}$ Para avaliar a correlação entre a altura, IMC e o tempo de sucção, foi utilizado o coeficiente de correlação de Spearman (rho). Os resultados são apresentados como média, desvio-padrão (DP) e coeficiente de correlação de Spearman. Foram considerados significantes os resultados com $\mathrm{p}<0,05$.
O projeto da investigação foi aprovado pela Comissão de Normas Éticas do Hospital das Clínicas da Faculdade de Medicina de Ribeirão Preto da Universidade de São Paulo, de acordo com o processo HCRP $\mathrm{n}^{\mathrm{o}}$ : 13.899/2010. Todos os voluntários assinaram o Termo de Consentimento Livre e Esclarecido.

\section{RESULTADOS}

O tempo de sucção foi menor nos jovens quando comparados com os idosos (tabela 1, $\mathrm{p}<0,01$ ), tanto com o canudo de $6 \mathrm{~mm}$ (figura 1), quanto com o canudo de $3 \mathrm{~mm}$ (figura 2). Com o canudo de $6 \mathrm{~mm}$ o tempo de sucção dos jovens foi de 2,96 (1,10)s e dos idosos 5,89 (2,09)s, e com o canudo de $3 \mathrm{~mm}$ o tempo foi de 4,63 $(1,43) \mathrm{s}$ nos jovens e de 8,69 (2,65)s nos idosos.

Tabela 1 - Idade, peso, altura e índice de massa corporal (IMC) dos indivíduos incluídos, e resultados do tempo de sucção medidos com o canudo de menor diâmetro (3 mm) e com o canudo de maior diâmetro (6 mm). Os resultados são apresentados como média e desvio-padrão. Ribeirão Preto, SP, 2010-2011.

\begin{tabular}{|c|c|c|c|c|}
\hline & \multicolumn{2}{|c|}{$\begin{array}{l}\text { JOVENS } \\
(\mathrm{N}=90)\end{array}$} & \multicolumn{2}{|c|}{$\begin{array}{l}\text { IDOSOS } \\
(\mathrm{N}=90)\end{array}$} \\
\hline & $\begin{array}{l}\text { HOMENS } \\
(\mathrm{N}=45)\end{array}$ & $\begin{array}{l}\text { MULHERES } \\
(\mathrm{N}=45)\end{array}$ & $\begin{array}{l}\text { HOMENS } \\
(\mathrm{N}=45)\end{array}$ & $\begin{array}{l}\text { MULHERES } \\
\quad(\mathrm{N}=45)\end{array}$ \\
\hline Idade (anos) & $23,9(2,9)$ & $23,1(2,7)$ & $72,5(5,6)$ & $72,4(5,8)$ \\
\hline Peso (kg) & $75,6(9,9)$ & $61,8(10,2)$ & $73,3(12,5)$ & $59,7(7,8)$ \\
\hline Altura (m) & $1,77(0,06)$ & $1,63(0,05)$ & $1,69(0,04)$ & $1,57(0,06)$ \\
\hline IMC $\left(\mathrm{kg} / \mathrm{m}^{2}\right)$ & $24,2(2,8)$ & $23,1(3,3)$ & $25,5(4,3)$ & $24,1(2,9)$ \\
\hline $\begin{array}{c}\text { Duração (s) } \\
\text { Diâmetro } 3 \mathrm{~mm}\end{array}$ & $4,36(1,41)^{*}$ & $4,80(1,51)^{*}$ & $7,22(2,45)^{* *}$ & $9,26(2,87)$ \\
\hline $\begin{array}{c}\text { Duração (s) } \\
\text { Diâmetro } 6 \mathrm{~mm}\end{array}$ & $2,65(1,01)^{*}$ & $3,10(1,18)^{*}$ & $5,16(2,13)$ & $5,95(2,03)$ \\
\hline
\end{tabular}

$* \mathrm{p}<0,01$ vs idosos; ${ }^{* *} \mathrm{p}=0.01$ vs mulheres idosas; $\mathrm{N}=$ número; $\mathrm{s}=$ segundos. 


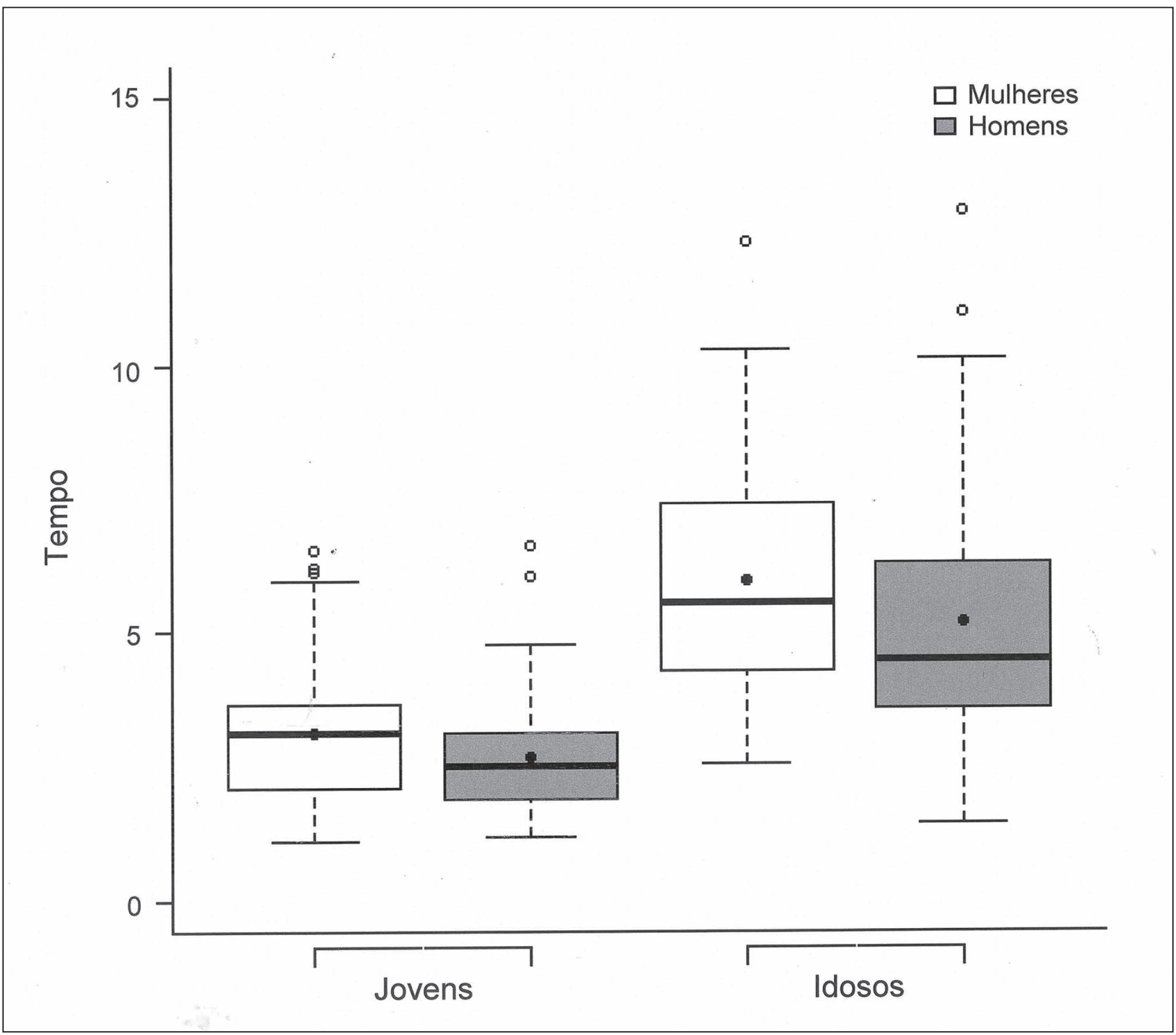

Figura 1 - Box-plot do tempo de sucção, em segundos, do volume de $40 \mathrm{ml}$ de água através de canudo com $6 \mathrm{~mm}$ de diâmetro, em homens e mulheres, jovens e idosos. As caixas representam os percentuais $25 \%$ e $75 \%$ da distribuição. Dentro da caixa o traço representa a mediana e o ponto representa a média. p<0,05 jovens vs idosos. Ribeirão Preto, SP, 2010-2011.

Houve diferença entre os sexos somente entre os idosos, com tempo de sucção maior entre as mulheres se comparado ao tempo dos homens, sendo significativa quando a avaliação foi realizada com o canudo de menor diâmetro (figura 2, $\mathrm{p}=0,01$ ). Com o canudo de $6 \mathrm{~mm}$, o tempo de sucção nas mulheres foi de 4,73(1,61)s e nos homens, 4,12(1,58)s; e com o canudo de 3 $\mathrm{mm}$, o tempo foi de $7,28(2,20) \mathrm{s}$ nas mulheres e $6,04(1,94)$ s nos homens.

A altura não se correlacionou significativamente com o tempo de sucção (tabela 2). Em mulheres jovens, o IMC se correlacionou positivamente com o tempo de sucção, com os canudos de $3 \mathrm{~mm}$ e de $6 \mathrm{~mm}$ (figura 3). 


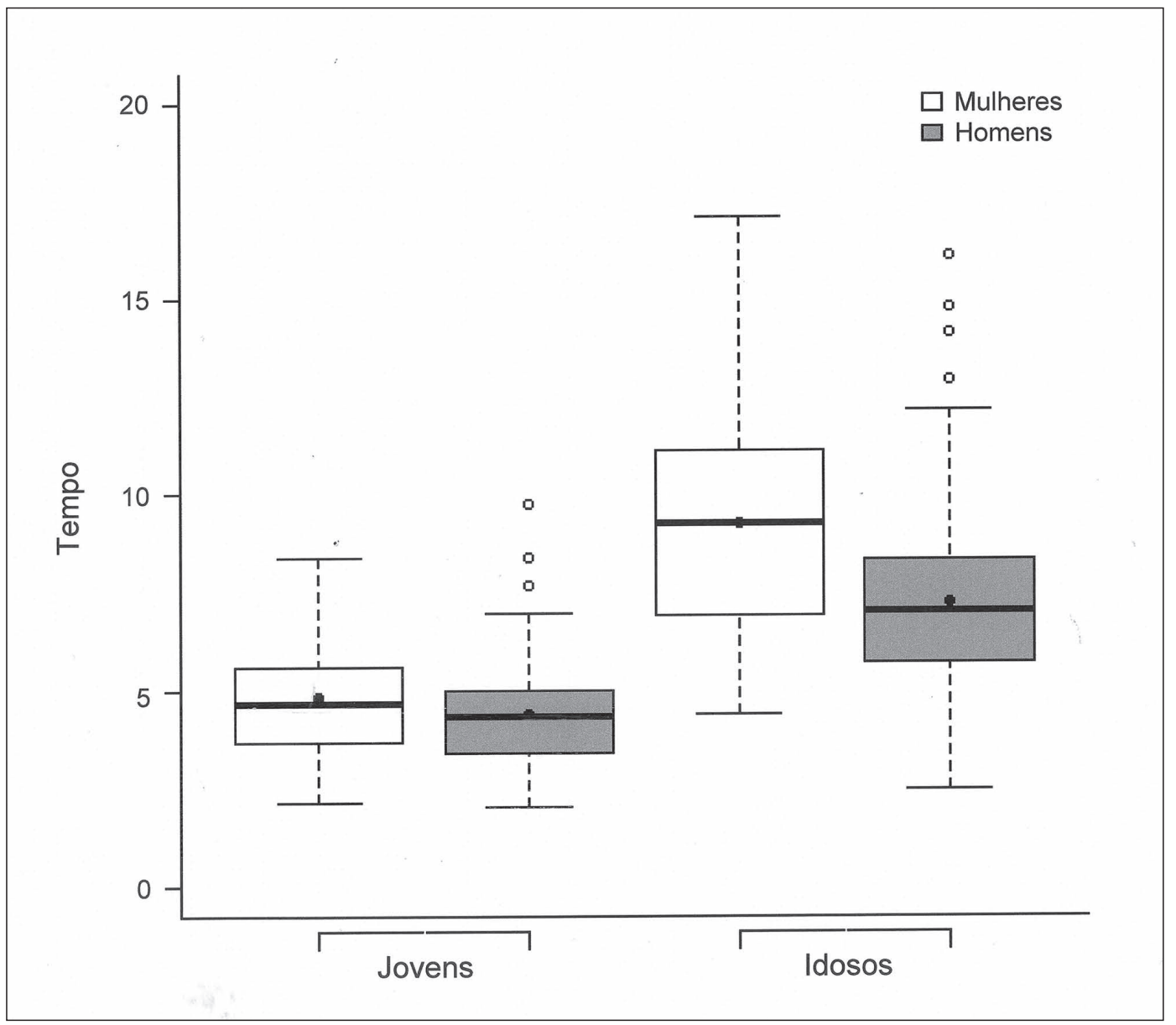

Figura 2 - Box-plot do tempo de sucção, em segundos, do volume de $40 \mathrm{ml}$ de água através de canudo com $3 \mathrm{~mm}$ de diâmetro, em homens e mulheres, jovens e idosos. As caixas representam os percentuais $25 \%$ e $75 \%$ da distribuição. Dentro da caixa, o traço representa a mediana e o ponto representa a média. $\mathrm{p}<0,05$ jovens vs idosos $\mathrm{p}=0,01$ homens idosos vs mulheres idosas. Ribeirão Preto, SP, 2010-2011.

Tabela 2 - Coeficiente de correlação de Spearman (rho) entre a altura e índice de massa corporal com o tempo de sucção de água com canudo de $3 \mathrm{~mm}$ e canudo de $6 \mathrm{~mm}$, em jovens e idosos, homens e mulheres. Ribeirão Preto, SP, 2010-2011.

\begin{tabular}{ccccccccc}
\hline & \multicolumn{4}{c}{ JOVENS } & \multicolumn{5}{c}{ IDOSOS } \\
\cline { 2 - 9 } & \multicolumn{2}{c}{ HOMENS } & \multicolumn{2}{c}{ MULHERES } & \multicolumn{2}{c}{ HOMENS } & \multicolumn{2}{c}{ MULHERES } \\
\cline { 2 - 9 } & rho & $\mathrm{p}$ & rho & $\mathrm{p}$ & rho & $\mathrm{p}$ & rho & $\mathrm{p}$ \\
\hline ALTURA & & & & & & & & \\
$3 \mathrm{~mm}$ & 0,14 & 0,36 & 0,11 & 0,49 & $-0,14$ & 0,35 & 0,28 & 0,07 \\
$6 \mathrm{~mm}$ & $-0,19$ & 0,21 & 0,13 & 0,41 & $-0,11$ & 0,46 & $-0,28$ & 0,06 \\
IMC & & & & & & & & \\
$3 \mathrm{~mm}$ & $-0,18$ & 0,24 & 0,35 & 0,02 & 0,13 & 0,44 & $-0,09$ & 0,55 \\
$6 \mathrm{~mm}$ & 0,00 & 0,99 & 0,32 & 0,03 & $-0,04$ & 0,78 & $-0,13$ & 0,40 \\
\hline
\end{tabular}

$\mathrm{p}=$ valor de $\mathrm{p}$ (probabilidade de a hipótese nula ser verdadeira). 


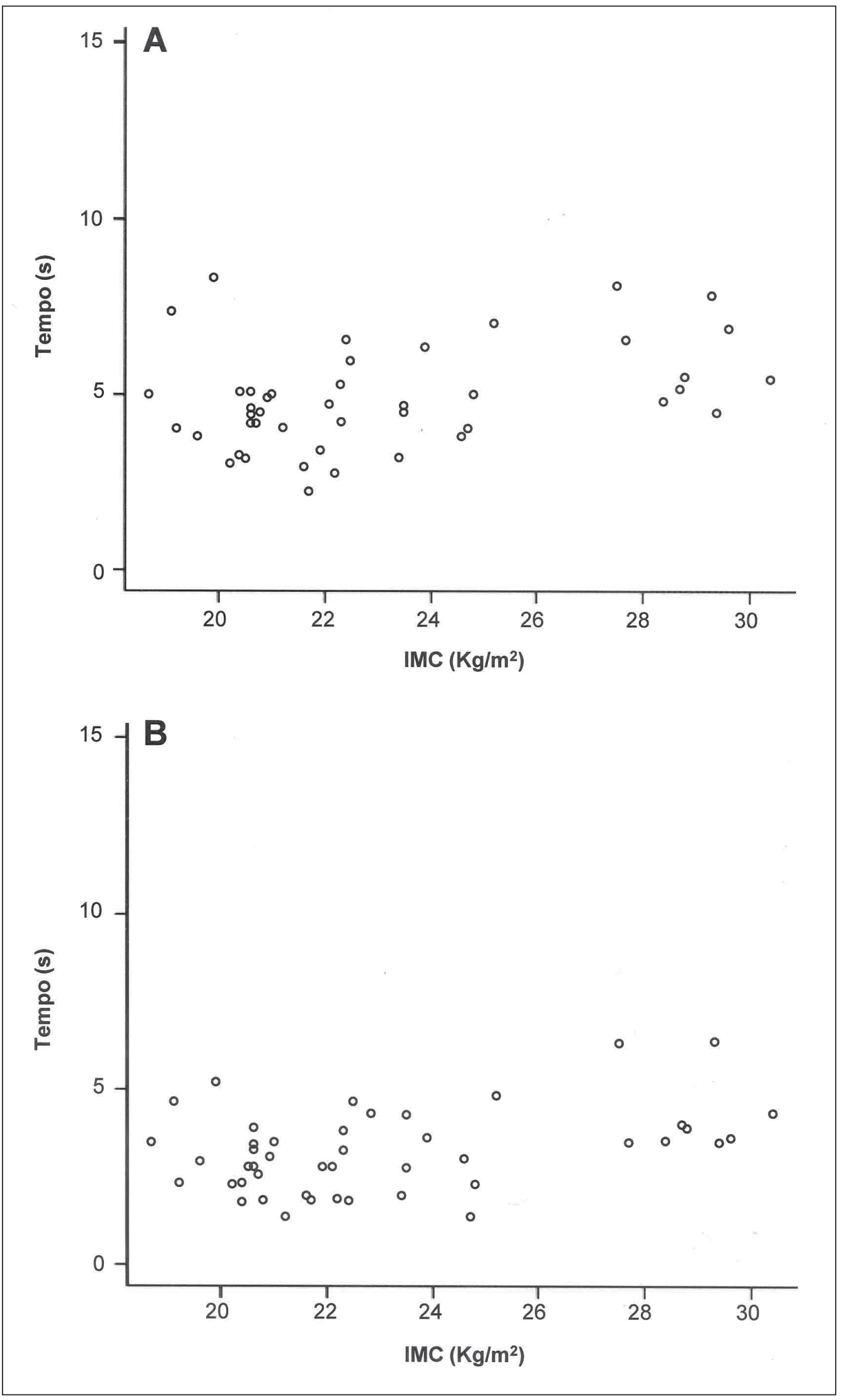

Figura 3 - Correlação entre o índice de massa corporal (IMC) e o tempo de sucção com o canudo de $3 \mathrm{~mm}$ (A) e de $6 \mathrm{~mm}$ (B) em mulheres jovens. A - Índice de correlação de Spearman rho: 0,35 $(\mathrm{p}=0,02) ; \mathrm{B}$ - Índice de correlação de Spearman rho: 0,32 ( $\mathrm{p}=0,03)$. Ribeirão Preto, SP, 2010-2011. 
A repetição do teste não encontrou diferenças entre os resultados obtidos nos quatro momentos em que ele foi realizado (tabela 3).

Tabela 3 - Resultados do tempo de sucção com a repetição do teste, realizado com canudo de maior e de menor diâmetro (segundos). Ribeirão Preto, SP, 2010-2011.

\begin{tabular}{|c|c|c|c|c|c|c|}
\hline \multirow[b]{2}{*}{ TESTE } & \multicolumn{3}{|c|}{ DIÂMETRO 3mm } & \multicolumn{3}{|c|}{ DIÂMETRO 6mm } \\
\hline & MÉDIA (DP) & IC 95\% & MEDIANA & MÉDIA (DP) & IC 95\% & MEDIANA \\
\hline 1 & $4,51(1,86)$ & $3,82-5,20$ & 4,05 & $2,36(1,25)$ & $1,89-2,82$ & 2,26 \\
\hline 2 & $4,53(2,05)$ & $3,76-5,30$ & 3,96 & $2,29(1,27)$ & $1,81-2,76$ & 1,97 \\
\hline 3 & $4,31(1,71)$ & $3,67-4,95$ & 3,99 & $2,37(1,09)$ & $1,97-2,78$ & 2,16 \\
\hline 4 & $4,40(1,82)$ & $3,72-5,08$ & 3,69 & $2,41(1,22)$ & $1,95-2,86$ & 1,84 \\
\hline
\end{tabular}

$\mathrm{IC}=$ intervalo de confiança; $\mathrm{DP}=$ desvio-padrão.

\section{DISCUSSÃO}

Observamos diferença significativa no tempo de sucção entre jovens e idosos, sendo maior para os idosos do que para os jovens. A diferença entre sexos foi significativa somente entre os idosos.

A sucção é controlada por um gerador de padrão central, isto é, uma rede neural localizada na formação reticular, de onde são enviados impulsos motores para a realização da função, que são modulados pela informação periférica. ${ }^{2}$

Com o método de sucção e deglutições únicas e repetitivas, observou-se que, em comparação a adultos jovens, os idosos apresentam menor pico de pressão de sucção, menor tempo de sucção, menor volume do bolo sugado e maior tempo de trânsito orofaríngeo, demonstrando haver efeito da idade na sucção e deglutição. ${ }^{23}$

Por meio do teste de ingestão de água em indivíduos saudáveis, foi observado que o volume por deglutição e o fluxo de deglutição foram maiores nos homens do que nas mulheres, e o intervalo entre as deglutições foi menor para os homens do que para as mulheres. E ainda, o intervalo de tempo entre as deglutições e o fluxo de deglutição diminui com o envelhecimento. ${ }^{14}$
Utilizando como método o mesmo teste, observou-se o mesmo efeito do sexo, ${ }^{6,19}$ e que o envelhecimento causa diminuição do fluxo de ingestão e do volume em cada deglutição. ${ }^{19}$

Ao contrário dos resultados do presente estudo, foi observado que a influência do sexo na deglutição é mais intensamente observada no grupo de jovens, e a influência da idade mais intensamente observada entre os homens. ${ }^{19} \mathrm{Com} \mathrm{o}$ método videofluoroscópico, a duração do trânsito total entre a boca e a transição faringoesofágica, de bolos líquido e pastoso, foi mais longa entre as mulheres do que entre os homens. ${ }^{10}$

A atividade eletromiográfica dos músculos labiais é maior quando se utiliza o canudo para ingestão de líquido do que com o uso de colher ou do copo. ${ }^{29} \mathrm{O}$ diâmetro do canudo determina o volume do bolo obtido na sucção, sendo que quanto menor o diâmetro do canudo, maior a atividade muscular. ${ }^{24}$

A média da contração muscular, medida pela eletromiografia de superfície, dos músculos masseter, infra-hióideos e submandibulares durante a deglutição em indivíduos adultos saudáveis, é maior em homens do que em mulheres, e diferença de um ano na idade causa 
diminuição de cerca de 0,99 vezes na intensidade da contração muscular, ou seja, quanto maior a idade, menor a intensidade de contração muscular. $^{30}$ Este fato está relacionado ao comprometimento muscular em consequência do envelhecimento. A perda da atividade eletromiográfica dos músculos orais justifica os resultados obtidos. Essa perda parece ser maior nas mulheres do que nos homens, mas outros estudos não encontraram efeitos na força máxima da língua relacionada ao envelhecimento.

Estudo realizado em ratos descreve que a força máxima da língua e fatigabilidade não são significativamente alteradas em animais idosos, mas o envelhecimento é associado com o aumento no tempo para atingir a força máxima e com longo intervalo de recuperação. ${ }^{31}$ Outro estudo, realizado em mulheres saudáveis, concluiu que o envelhecimento não altera a força da língua e não altera a amplitude do sinal eletromiográfico, medido na região submentoniana, e que mulheres de mais idade atingem mais lentamente a pressão máxima, consequente ao contato anterior da língua com o palato. ${ }^{32}$ Os autores concluíram que, apesar das alterações da deglutição observadas com o envelhecimento, mulheres idosas podem ter deglutições com pressão da língua e amplitude do sinal eletromiográfico dos músculos da região submentoniana similares a de mulheres jovens. ${ }^{32}$

Considerando essas duas publicações, ${ }^{31,32}$ podemos entender os resultados obtidos como consequentes ao tempo necessário para atingir a força máxima de sucção, e não à diminuição desta força máxima. Esta hipótese necessita de outras investigações para ser esclarecida.

Outro fator que deve ser considerado no tempo de sucção é que as possíveis deglutições podem ter influência no tempo total, e há resultados demonstrando que idosos demoram mais para ingerir um mesmo volume líquido do que jovens. ${ }^{20} \mathrm{Em}$ mulheres jovens, houve correlação positiva, embora fraca, entre IMC e tempo de sucção, significando que quanto maior o IMC, mais longo o tempo de sucção. É possível que o peso tenha influência na força oral de sucção, hipótese que necessita mais investigações.
É descrito que a obesidade não altera a força do músculo da língua em ratos. ${ }^{33} \mathrm{Não}$ estudamos indivíduos com IMC acima de $35 \mathrm{~kg} / \mathrm{m}^{2}$ porque a obesidade dessas pessoas poderia retirar desses sujeitos a característica de normalidade e alterar a sucção. Pelo que encontramos nas mulheres jovens, esta possibilidade deve ser verdadeira. É possível também que a menor estatura das mulheres $(1,60 \mathrm{~m})$, em relação aos homens $(1,76 \mathrm{~m})$, tenha influenciado a análise da influência do sexo; entretanto, a existência de influência da estatura nesta função ainda não é bem conhecida.

Os resultados encontrados indicam que a força de sucção em pessoas idosas, principalmente em mulheres, está diminuída em relação aos jovens. Este conhecimento é importante para aqueles que trabalham como cuidadores de pessoas idosas. Sabendo da menor eficiência da sucção nesta população, é esperado que idosos tenham maior tempo para ingerir uma refeição quando este meio é utilizado, ou seja, refeição líquida ingerida por canudo. Aspirar maior volume em menor tempo tem influência na percepção do gosto do alimento, ${ }^{5}$ importante para pessoas com dificuldades na deglutição, mas aspirar maior volume em menor tempo pode não ser possível em pessoas idosas.

A investigação tem algumas limitações, como a possível ocorrência de deglutição durante a sucção, e a possível interrupção da sucção pela respiração, o que pode ter influenciado o tempo de sucção. No entanto, as duas situações podem estar presentes durante o processo fisiológico de ingestão líquida.

\section{CONCLUSÃO}

Concluímos que o tempo de sucção de volume líquido por meio de um canudo é menor na pessoa jovem do que na pessoa idosa, independentemente do sexo. O aumento do tempo de sucção com o envelhecimento parece ser mais acentuado nas mulheres, quando comparadas com o aumento do tempo nos homens. Canudo de menor diâmetro causa maior duração da sucção. Não há influência da altura. Em mulheres jovens, o tempo de sucção tem correlação positiva com o índice de massa corporal. 


\section{REFERÊNCIAS}

1. Gouch SA. Infantile sexuality revisited: the agony and extasy of the mother-infant couple. J Am Assoc Psychol 1991;19(2):254-70.

2. Whohlert AB, Goffman L. Human perioral muscle activation patterns. J. Speech Lang Hear Res 1994;37(5):1032-40.

3. Iderilha PN, Limongi, SCO. Avaliação eletromiográfica da sucção em bebês com síndrome de Down. Rev Soc Bras Fonoaudiol 2007;12(3):174-83.

4. Amaizu N, Shulman RJ, Schandler RJ, Lou C. Maturation of oral feeding skills in preterm infants. Acta Paediatr 2008;97(1):61-7.

5. Lawless HT, Bender S, Oman C, Pelletier C. Gender, age, vessel size, cup vs. straw sipping, and sequence effects on sip volume. Dysphagia 2003;18(3):196-202.

6. Alves LM, Cassiani Rde A, Santos CM, Dantas RO. Gender effect on the clinical measurement of swallowing. Arq Gastroenterol 2007;44(3):227-9.

7. Cook IJ, Weltman MD, Wallace K, Shaw DW, Mckay E, Smart RC, et al. Influence of aging on oral-pharyngeal bolus transit and clearance during swallowing: scintigraphic study. Am J Physiol 1994;266(6):G972-7.

8. Dantas RO, Ferriolli E, Souza MA. Gender effects on esophageal motility. Braz J Med Biol Res 1998;31(4):539-44.

9. Dantas RO, Alves LMT, Cassiani Rde A. Gender differences in proximal esophageal contractions. Arq Gastroenterol 2009;46(4):284-7.

10. Dantas RO, Cassiani Rde A, Santos CM, Gonzaga GC, Alves LM, Mazin SC. Effect of gender on swallow event duration assessed by videofluoroscopy. Dysphagia 2009;24(3):280-4.

11. Dantas RO, Alves LM, Dalmazo J, Santos CM, Cassiani Rde A, Nascimento WV. Effect of age on proximal esophageal response to swallowing. Arq Gastroenterol 2010;47(4):339-43.

12. Ferriolli E, Dantas RO, Oliveira RB, Braga FJ. The influence of aging on oesophageal motility after ingestion of liquids with different viscosities. Eur J Gastroenterol Hepatol 1996;8(8):793-8.

13. Gluckman A. Sexual dimorphism in human and mammalian biology and pathology. New York: Academic Press; 1981.

14. Hughes TA, Wiles CM. Clinical measurement of swallowing in healthy and in neurogenic dysphagia. QJM 1996;89(2):109-16.
15. Kang BS, Oh BM, Kim IS, Chung SG, Kim SJ, Han TR. Influence of aging on movement of the hyoid bone and epiglottis during normal swallowing: a motion analysis. Gerontology 2010;56(5):474-82.

16. Kurosu A, Logemann JA. Gender effects on airway closure in normal subjects. Dysphagia 2010;25(4):284-90.

17. Lang IM. Brain stem control of the phases of swallowing. Dysphagia 2009;24(3):333-48.

18. Logemann JA, Pauloski BR, Rademaker AW, Kahrilas PJ. Oropharyngeal swallow in younger and older women: videofluoroscopic analysis. J Speech Lang Hear Res 2002;45(3):434-45.

19. Dantas RO, Alves LM, Santos CM, Cassiani Rde A.. Possible interaction of age and gender on human swallowing behavior. Arq Gastroenterol 2011;48(3):195-8.

20. Nascimento WV, Cassiani Rde A, Dantas RO. Gender effect on oral volume capacity . Dysphagia 2011;27(3):384-9.

21. Shaker R, Ren J, Podyrsan B, Dodds WJ, Hogan WJ, Kern M, et al. Effect of aging and bolus variables on pharyngeal and upper esophageal sphincter motor function. Am J Physiol 1993;264(3):G427-32.

22. Van Herwaarden MA, Katz PO, Gideon RM, Barrett J, Castell JA, Achem S, et al. Are manometric parameters of the upper esophageal sphincter and pharynx affected by age and gender? Dysphagia 2003;18(3):211-7.

23. Nilsson H, Ekberg O, Olsson R, Hindfelt B. Quantitative aspects of swallowing in an elderly nondysphagic population. Dysphagia 1996;11(3):180-4.

24. Chandler C, et al. Effect of straw diameter on bolus volume and muscle activity. Dysphagia 2009:24(4):471 (Abstract)

25 Bennett JW, Van Lieshout PH, Steele CM. Tongue control for speech and swallowing in healthyyounger and older subjects. Int J Orofacial Myology 2007;33:5-18.

26. Associação Brasileira para o Estudo da Obesidade e da Síndrome Metabólica. [homepage na internet]. Calcule seu IMC (Índice de Massa Corporal) [acesso em 20 Out 2011]. Disponível em: http://www.abeso. org.br/calcule-seu-imc.shtml

27. Schall R. Estimation in generalized linear models with random effects. Biometrika 1991;78(4):719-27.

28. SAS Institute Inc., SAS/STAT® User's Guide, Version 9, Cary, NC: SAS Institute Inc., 1999. 
29. Murray KA, Larson CR, Logemann JA.

Electromyographic response of the labial muscles during normal liquid swallows using a spoon, a straw, and a cup. Dysphagia 1998;13(3):160-6.

30. Siqueira LA, Angelis EC, Vartanian JG. Contração da musculatura massetérica, infra-hióidea e submandibular durante a deglutição considerando gênero e idade. 2011 [acesso em 20 Out 2011]. Disponível em: http://www. sbfa.org.br/portal/suplementorsbfa .
31. Ota F, Connor NP, Konopacki R. Alterations in contractile properties of tongue muscle in old rats. Ann Otol Rhinol Laryngol 2005;114(10):799-803.

32. Yeates EM, Steele CM, Pelletier CA. Tongue pressure and submental electromyography measures during noneffortful and effortful saliva swallows in healthy women. Am J Speech Lang Pathol 2010;19(3):274-81.

33. Ray AD, Farkas GA, Pendergast DR. In-situ mechanical characteristics of the tongue are not altered in the obese Zucker rat. Sleep 2009;32(7):957-61. 